\title{
A nonlinear blind identification approach to modeling of diabetic patients
}

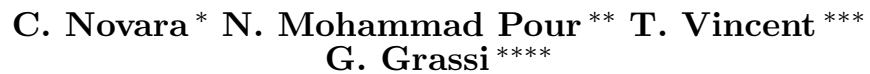 \\ *Politecnico di Torino, Italy (e-mail: carlo.novara@polito.it). \\ ** University of Southampton, UK (e-mail: \\ nima.mohammadpour@soton.ac.uk). \\ *** Colorado School of Mines, USA (e-mail: tvincent@mines.edu) \\ **** Città della Salute e della Scienza, Torino, Italy (e-mail: \\ giorgio.grassi@unito.it)
}

\begin{abstract}
Modeling, simulation and control have become effective tools for the treatment of type 1 diabetic patients in the last decades. The availability of reliable models able to predict and/or simulate the behavior of diabetic patients is thus fundamental in this context. Several models, based on first principles or black-box approaches, have been proposed to fulfill this need. However, a common problem to these approaches is that they are not able to recover or to systematically account for the various unmeasured signals which affect a diabetic patient (e.g. food, physical activity, emotions, etc.). In this paper, we propose a blind identification approach, which allows us to derive accurate models of type 1 diabetes patients and to efficiently recover the unmeasured input signals. A simulated example, regarding identification of the blood glucose concentration in type 1 diabetes patients, is presented to demonstrate the effectiveness of the proposed approach.
\end{abstract}

Keywords: Diabetes modeling and control, blind identification, nonlinear systems.

\section{INTRODUCTION}

In the last decades, there has been a growing interest in modeling, simulation, prediction and control of diabetes mellitus type 1 (DMT1), with the aim of improving blood glucose regulation so to avoid the complications resulting from hyperglycemia, Bequette (2012); Harvey et al. (2010); Renard et al. (2013). Control of diabetes is accomplished by planning and applying the most proper therapy strategy for a given patient. This strategy can be either manual or automated. If automated, it is called "artificial pancreas" and represents an extremely promising technology for the future, Cobelli et al. (2011); Lee et al. (2013). In both cases, control of the blood glucose concentration has a fundamental role in terms of patient wellness and integrity of organs that may be damaged due to DMT1.

Control of the blood glucose concentration implies the availability of reliable models able to predict and/or simulate the behavior of metabolic control in diabetes. Different models and modeling techniques have been proposed in the literature, where physiology equations are used to describe the glucose and insulin kinetics in the body, Ajmera et al. (2013); Dube et al. (2013). However, these models are in general not extremely accurate as their equations do not take into account all the dynamics, parameters and disturbances involved in the patient system. Black-box models have the potential of contributing to the solution of this problem, Cobelli et al. (2009); Gonzalez-Olvera et al. (2009); Stahl and Johansson (2009); Finan et al. (2009). These models are directly identified from experimental data and, for this reason, are in general more accurate than models based on the first-principles. Another important advantage of input-output techniques is that they easily allow the construction of personalized patient models, which can be more effective for simulation, control and analysis of DMT1 than models providing an average description of the patient population.

In the diabetes context, a relevant problem common to both first-principle and black-box approaches is that a patient is a system affected by unmeasured (or not easily measurable) inputs (e.g. food, physical activity, emotions, etc.), and the techniques frequently used for model identification are in general not able to recover or to account for such unmeasured signals. Statistical techniques may be used to estimate the unmeasured inputs, but recovery is usually possible (asymptotically) only when these signals are white noises. However, inputs such as food, physical activity, emotions, etc. are far from being white processes and thus can be hardly recovered using standard techniques.

Indeed, modeling of a diabetes patient can be seen as a blind identification problem: not only the patient system has to be identified but also some of the system input signals, Abed-Meraim et al. (1997). For this reason, blind identification problems are in general significantly more difficult than the standard identification problems, where all the inputs are assumed to be measured (except that some noise with given statistical or boundedness properties). A number of approaches to blind identification have been developed for linear systems, see e.g. Abed-Meraim et al. (1997); Ohlsson et al. (2013). Several approaches can also be found for nonlinear systems, but these mainly regard Hammerstein, Bai and Minyue (2002), Wiener, Wills et al. (2011), bilinear, Kalouptsidis et al. (2003), and Volterra, Mileounis and Kalouptsidis (2009), systems. To the best of the authors' knowledge, no solutions are available for the problem considered here, involving general nonlinear systems. 
In this paper, we propose a novel black-box model identification approach for general nonlinear systems, based on $\ell_{1}$ sparsification, allowing us to derive accurate models of DMT1 patients and to recover the unmeasured input signals (called also disturbances in the following). More precisely, the approach allows us to recover a surrogate disturbance signal which describes the effect of all unknown inputs acting on the patient system.

A simulated example, regarding identification and prediction of the blood glucose concentration in DMT1 patients, is presented to demonstrate the effectiveness of the proposed blind identification approach.

\section{BLIND IDENTIFICATION OF NONLINEAR SYSTEMS}

\subsection{Problem formulation}

Consider a nonlinear discrete-time system in regression form:

$$
\begin{array}{r}
y_{k}=g^{o}\left(y_{k-1}, y_{k-2}, \ldots, y_{k-n},\right. \\
u_{k}, u_{k-1}, \ldots, u_{k-n}, \\
\left.w_{k}, w_{k-1}, \ldots, w_{k-n}\right)
\end{array}
$$

where $y_{k} \in \mathbb{R}$ is the output, $u_{k} \in \mathbb{R}^{n_{u}}$ and $w_{k} \in \mathbb{R}^{n_{w}}$ are external inputs, and $n$ is the system order. Suppose that $u_{k}$ and $y_{k}$ can be measured, $w_{k}$ cannot be measured, and the function $g^{o}$ is unknown. Let a set of noise-corrupted measurements

$$
D^{I} \doteq\left\{\tilde{y}_{k}, \tilde{u}_{k}\right\}_{k=1}^{L}
$$

be available, where the tilde denotes the measured values of the variables. In this paper, we consider the problem of deriving a reliable model of the system (1), together with some estimate of the unknown input $w_{k}, k=1, \ldots, L$.

This problem can be seen as a blind identification problem, since not only the system has to be identified but also some of the system input signals, Abed-Meraim et al. (1997). A further difficulty besides the fact that $w_{k}$ is unknown is that it appears nonlinearly in the system equation (1). Its estimation could thus require to solve a very hard nonconvex optimization problem. In this paper, we propose a solution for general nonlinear systems where, in order to deal with convex optimization, we estimate a surrogate $d_{k}^{o}$ of $w_{k}$, which describes the effect of $w_{k}$ on the system output.

Assume that the function $g^{o}$ is differentiable with respect to $w_{k}, \ldots, w_{k-n}$ and define

$$
r_{k} \doteq\left(y_{k-1}, \ldots, y_{k-n}, u_{k}, \ldots, u_{k-n}\right) \in \mathbb{R}^{m} .
$$

where $m=n+(n+1) n_{u}$. From the Mean Value Theorem it follows that, for some $\bar{w}_{k}, \ldots, \bar{w}_{k-n} \in \mathbb{R}^{n_{w}}$,

$$
y_{k}=g^{o}\left(r_{k}, 0, \ldots, 0\right)+\sum_{i=0}^{n} q_{k, i} \cdot w_{k-i}
$$

where $q_{k, i} \doteq \frac{\partial g^{o}\left(r_{k}, \bar{w}_{k}, \ldots, \bar{w}_{k-n}\right)}{\partial w_{k-i}}$ is the gradient of $g^{o}$ with respect to $w_{k-i}$ computed in $\left(\bar{w}_{k}, \ldots, \bar{w}_{k-n}\right)$, and $\cdot$ denotes the dot product. The system (1) can thus be written as

$$
y_{k}=f^{o}\left(r_{k}\right)+\sum_{i=0}^{n} q_{k, i} \cdot w_{k-i}
$$

where $f^{o}\left(r_{k}\right) \doteq g^{o}\left(r_{k}, 0, \ldots, 0\right)$. Consider now the mean over $i$ of $q_{k, i}: \bar{q}_{k} \doteq \frac{1}{n+1} \sum_{i=0}^{n} q_{k, i}$. Defining $d_{k-i}^{o} \doteq \bar{q}_{k} w_{k-i}$, from (3), we obtain the following system representation:

$$
y_{k}=f^{o}\left(r_{k}\right)+\sum_{i=0}^{n} d_{k-i}^{o}+e_{k}^{o}
$$

where $e_{k}^{o} \doteq \sum_{i=0}^{n}\left(q_{k, i}-\bar{q}_{k}\right) w_{k-i}$ is a noise and $d_{k}^{o}$ is an unknown input which describes the effect on the system output of $w_{k}$ in (1).

The problem considered in this paper is the following:

Problem: From the data set (2), find reliable estimates $\hat{f}$ and $\hat{d}=\left(\hat{d}_{1}, \ldots, \hat{d}_{L}\right)$ of the regression function $f^{o}$ and the input sequence $d^{o}=\left(d_{1}^{o}, \ldots, d_{L}^{o}\right)$, respectively.

\subsection{Basic assumptions}

According to (4), the $L$ measured data in (2) can be described as

$$
\tilde{y}_{k}=f^{o}\left(\tilde{r}_{k}\right)+\sum_{i=0}^{n} d_{k-i}^{o}+e_{k}, k=n+1, \ldots, L
$$

where $e_{k}$ is a noise accounting for $e_{k}^{o}$ in (4) and for the measurement noises corrupting $y_{k}$ and $r_{k}$.

Without assumptions on the regression function $f^{o}$, on the input $d_{k}^{o}$ and on the noise $e_{k}$, the blind identification problem is ill-posed, in the sense that infinitely many functions, input sequences and noise sequences exist which are consistent with the measured data (i.e. satisfy equation $(5))$. In this paper, we consider the following assumptions.

Assumption on $e_{k}$. The noise sequence $e=\left(e_{n+1}, \ldots, e_{L}\right)$ is bounded as

$$
\|e\|_{2} \leq \varepsilon
$$

where $\|\cdot\|_{2}$ is the vector $\ell_{2}$-norm. This assumption is standard Milanese and Vicino (1991).

Assumption on $f^{o}$. The regression function $f^{o}$ is described by a linear combination of basis functions:

$$
f^{o}\left(r_{k}\right)=\sum_{i=1}^{N} a_{i}^{o} \phi_{i}\left(r_{k}\right)
$$

where $\phi_{i}: \mathbb{R}^{m} \rightarrow \mathbb{R}$ are basis functions and $a_{i}^{o} \in \mathbb{R}$ are coefficients. This assumption is standard in system identification Sjöberg et al. (1995).

Assumption on $d_{k}^{o}$. The input sequence $d^{o}$ admits a sparse representation:

$$
d_{k}^{o}=\sum_{i=1}^{M} b_{i}^{o} \psi_{i}(k), k=1, \ldots, L
$$

where $\psi_{i}: \mathbb{Z} \rightarrow \mathbb{R}$ are basis functions and the coefficient vector $b^{o}=\left(b_{1}^{o}, \ldots, b_{M}^{o}\right)$ is sparse, i.e. it has only a limited number of non-zero components. More precisely, $b^{o}$ has minimum $\ell_{0}$-quasi-norm over all vectors consistent with (5), (7), (6) and (8). The $\ell_{0}$-quasi-norm of a vector is defined as the number of its non-zero components. This assumption is widely used in signal processing and information theory, and relies on the idea that many signals in the real world can be represented by a limited number of basis functions Fuchs (2005); Tropp (2006); Donoho et al. (2006); Candes and Tao (2006)Donoho et al. (2006). Note that this assumption is not restrictive. Indeed, in practice, a "large" number of basis functions can be considered to form the expansion (8), including possibly different types of functions (e.g. Gaussian, wavelet, polynomial, trigonometric). The identification algorithm presented in Section 2.3 below will allow us to select, among this "large" set of functions, those which are more appropriate to describe the unknown sequence $d^{o}$. 


\subsection{Identification algorithm}

Based on assumptions (7) and (8), estimates of the unknown regression function $f^{o}$ and input $d_{k}^{o}$ are looked for, of the following form:

$$
\begin{aligned}
& \hat{f}\left(r_{k}\right)=\sum_{i=1}^{N} \hat{a}_{i} \phi_{i}\left(r_{k}\right) \\
& \hat{d}_{k}=\sum_{i=1}^{M} \hat{b}_{i} \psi_{i}(k)
\end{aligned}
$$

where $\hat{a}_{i}$ and $\hat{b}_{i}$ are parameters to be identified. In order to ensure consistency with the measured data (described by (5)) and assumption (6), these parameters must satisfy the following inequalities:

$$
\left|\tilde{y}_{k}-\sum_{i=1}^{N} \hat{a}_{i} \phi_{i}\left(\tilde{r}_{k}\right)+\sum_{j=0}^{n} \sum_{i=1}^{M} \hat{b}_{i} \psi_{i}(k-j)\right| \leq \varepsilon
$$

for $k=n+1, \ldots, L$, which can be written in the more compact matrix form

$$
\|\tilde{y}-\Phi a-\Omega b\|_{2} \leq \varepsilon
$$

where

$$
\begin{aligned}
& \tilde{y}=\left(\tilde{y}_{n+1}, \ldots, \tilde{y}_{L}\right) \in \mathbb{R}^{(L-n)} \\
& a=\left(a_{1}, \ldots, a_{N}\right) \in \mathbb{R}^{N} \\
& b=\left(b_{1}, \ldots, b_{M}\right) \in \mathbb{R}^{M} .
\end{aligned}
$$

The matrices $\Phi \in \mathbb{R}^{(L-n) \times N}$ and $\Omega \in \mathbb{R}^{(L-n) \times M}$ are defined as follows:

$$
\begin{aligned}
& \Phi \doteq\left[\begin{array}{ccc}
\phi_{1}\left(\tilde{r}_{n+1}\right) & \cdots & \phi_{N}\left(\tilde{r}_{n+1}\right) \\
\vdots & \ddots & \vdots \\
\phi_{1}\left(\tilde{r}_{L}\right) & \cdots & \phi_{N}\left(\tilde{r}_{L}\right)
\end{array}\right] \\
& \Psi \doteq\left[\begin{array}{ccc}
\psi_{1}(1) & \cdots & \psi_{M}(1) \\
\vdots & \ddots & \vdots \\
\psi_{1}(L) & \cdots & \psi_{M}(L)
\end{array}\right] \\
& \Lambda \doteq\left[\begin{array}{ccccccc}
1 & \cdots & 1 & 0 & \cdots & \cdots & 0 \\
0 & 1 & \cdots & 1 & 0 & \cdots & 0 \\
\vdots & \ddots & \ddots & \ddots & \ddots & \ddots & \vdots \\
0 & \cdots & \cdots & 0 & 1 & \cdots & 1
\end{array}\right]
\end{aligned}
$$

where the sequence $1 \cdots 1$ on each row of $\Lambda$ has length $n+1$. Without loss of generality, we assume that $\Phi$ and $\Omega$ have columns normalized in $\ell_{2}$-norm.

The idea is to look for parameter vectors $\hat{a}$ and $\hat{b}$ that, among all vectors consistent with the measured data and prior assumptions (i.e. all vectors which satisfy inequality (10)), give a maximally sparse representation of the disturbance signal $\hat{d}_{k}$. This task is accomplished by the following convex optimization problem:

$$
\begin{aligned}
(\hat{a}, \hat{b})= & \arg \min _{(a, b)}\|b\|_{1} \\
& \text { subject to }\|\tilde{y}-\Phi a-\Omega b\|_{2} \leq \varepsilon
\end{aligned}
$$

where the vector $\ell_{1}$-norm is used to maximize the sparsity of $\hat{b}$ (and thus of the disturbance representation).

The identified model is described by the following difference equation:

$$
\hat{y}_{k}=\hat{f}\left(\hat{r}_{k}\right)+\sum_{i=0}^{n} \hat{d}_{k-i}
$$

where $\hat{r}_{k} \doteq\left(\hat{y}_{k-1}, \ldots, \hat{y}_{k-n}, u_{k}, \ldots, u_{k-n}\right), \hat{f}$ and $\hat{d}_{k}$ are given by $((9))$, and the parameters $\hat{a}_{i}$ and $\hat{b}_{i}$ defining $\hat{f}$ and $\hat{d}_{k}$ are obtained from the optimization problem (12).
The choice of the basis functions $\phi_{i}$ and $\psi_{i}$ is clearly an important point of the identification process. In some cases of practical interest, the basis functions may be known a priori, see Novara $(2011,2012)$. In other cases, the basis functions are not known and their choice can be carried out considering the numerous options available in the literature (e.g. Gaussian, exponential, sigmoidal, wavelet, polynomial, trigonometric). Observation of the measured signals can eventually be used for the proper basis function choice, see the example presented in Section 3.

Conditions under which the minimization problem (12) is able to recover the unknown signal $d_{k}^{o}$ in (8), are given in Vincent and Novara (2013).

\section{IDENTIFICATION OF DIABETES SYSTEMS (SIMULATED DATA)}

\subsection{Description of the considered diabetic patient system}

The model shown in Figure 1, representing a type 1 diabetes patient has been considered in this example. The inputs of this model are the carbohydrate-based meal input and the insulin input function, the output is the blood glucose concentration (glycemic response). The state equations defining the model are the following:

$$
\begin{aligned}
\frac{d y(t)}{d t} & =-\left[p_{1}+\eta(t)\right] y(t)+p_{1} G_{b}+\frac{w(t)}{V_{g}} \\
\frac{d \eta(t)}{d t} & =-p_{2} \eta(t)+p_{3}\left[I(t)-I_{b}\right] \\
\frac{d I(t)}{d t} & =\frac{k_{a}}{V_{d}} I_{2}(t)-k_{e} I(t) \\
\frac{d I_{1}(t)}{d t} & =-k_{21} I_{1}(t)+u(t) \\
\frac{d I_{2}(t)}{d t} & =k_{21} I_{1}(t)-\left(k_{d}+k_{a}\right) I_{2}(t)
\end{aligned}
$$

where $y(t)$ is the blood glucose concentration (the system output), $I(t)$ is the blood insulin concentration, $\eta(t)$ is the insulin concentration in a remote compartment, $V_{g}$ is the volume distribution, $w(t)$ is the carbohydrate-based meal input (a system input), $I_{1}(t)$ is the subcutaneous insulin mass in the injection depot, $I_{2}(t)$ is the subcutaneous insulin mass proximal to plasma and $u(t)$ is the insulin input function (a system input). $p_{1}, p_{2}, p_{3}$ are individual subject parameters, $V_{d}$ is the plasma distribution volume, $k_{21}, k_{a}, k_{d}$, and $k_{e}$ are insulin pharmacokinetic parameters, $I_{b}$ is the basal blood insulin concentration and $G_{b}$ is the basal blood glucose concentration. The first two equations of (14), describing the glucose dynamics, have been taken from the Bergman model, Bergman et al. (1981); the last three equations of (14), describing the insulin kinetics, have been taken from the Shimoda model, Nucci and Cobelli (2000). The following parameter values have been assumed: $p_{1}=0.031 \mathrm{~min}^{-1}, p_{2}=0.012 \mathrm{~min}^{-1}$, $p_{3}=9.56 e^{-6} 1 / \mathrm{min}^{2} / \mu U / \mathrm{mL}, V_{g}=1.45 \mathrm{dL} / \mathrm{kg}, V_{d}=$ $0.2 \mathrm{~mL} / \mathrm{kg}, k_{21}=0.0166 \mathrm{~min}^{-1}, k_{a}=0.0133 \mathrm{~min}^{-1}$, $k_{d}=0.0033 \mathrm{~min}^{-1}, k_{e}=0.3 \mathrm{~min}^{-1}, I_{b}=0 \mu U / m L$ and $G_{b}=180 \mathrm{mg} / \mathrm{dL}$. In this simulated example, the model (14) represents the "true" patient system to be identified.

Note that the model (14) is not the most recent that can be found in the literature and may also be not sufficiently adequate to describe a real diabetes patient. However, the aim of this numerical example is to test our blind identification algorithm on a non trivial nonlinear system and thus the particular choice of the model used as the "true" system is not so relevant for this kind of test. A 


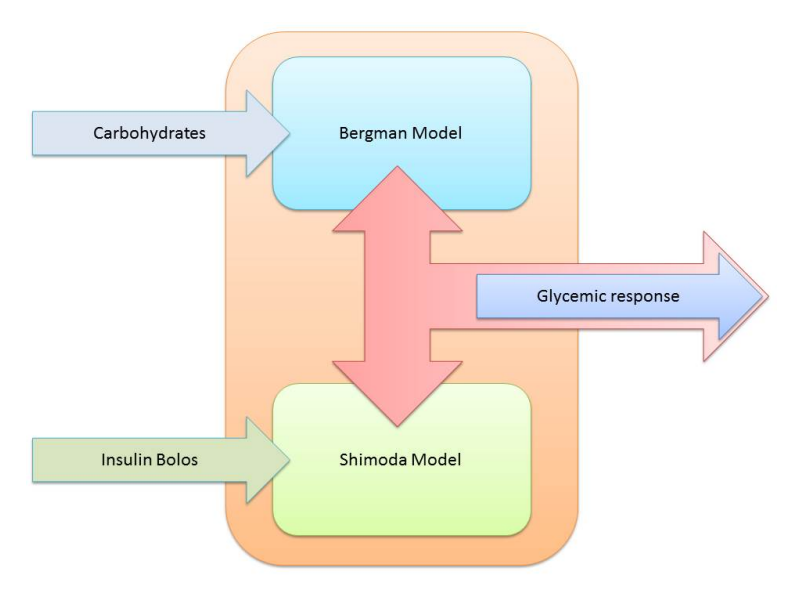

Fig. 1. Model of a DMT1 patient.

further aspect for which this example is of interest is that we can compare the estimated disturbance with the true one (this cannot be done in a real problem).

\subsection{Data generation and organization}

A simulation of the patient system (14) has been performed, where the insulin input has been taken from a set of experimental data, measured on a real patient. The meal input has been simulated as a superposition of exponentially decaying signals $w_{j}(t) j=1,2, \ldots$, where each contribution $w_{j}(t)$ represents a single meal. These signals are of the form

$$
w_{j}(t)= \begin{cases}0, & t<t_{j} \\ \left(t-t_{j}\right) e^{-0.6\left(t-t_{j}\right)}, & t \geq t_{j}\end{cases}
$$

where $t_{j}$ is the time at which the patient started to eat. The times $t_{j}$ have been realistically chosen in order to have an insulin injection a few minutes before a meal. The output signal (the blood glucose concentration) resulting from this simulation has been corrupted by a white noise, having a noise-to-signal standard deviation ratio of $3 \%$.

A set of 3000 data has been collected from this simulation, using a sampling time of 5 minutes. The set $D^{I} \doteq$ $\left\{\tilde{y}_{k}, \tilde{u}_{k}\right\}_{k=1}^{1500}$, composed of the first 1500 data, called the identification set, has been used for model identification. The set $D^{V} \doteq\left\{\tilde{y}_{k}, \tilde{u}_{k}\right\}_{k=1501}^{3000}$, composed of the remaining data, called the validation set, has been used for model validation.

\subsection{Model identification and meal input recovering}

A model of the form (13), called here BIM, has been identified on the identification set $D^{I}$ by means of the proposed algorithm (12), assuming the order $n=5$. Polynomial basis functions $\phi_{i}$ with degree ranging in the interval $[0,2]$ have been considered for the function $f^{\circ}$. Gaussian basis functions have been assumed for the disturbance $d^{o}$ :

$$
\psi_{i}(k)=e^{-\beta(k-l)^{2}}
$$

where $l=1, \ldots, 1500, \beta=0.05,0.2,0.5$ and, for each combination of $l$ and $\beta$ in (15), we assigned a progressive index $i=1, \ldots, M$, with $M=4500$. Both polynomial and Gaussian basis functions represent standard choices in system identification and signal processing. Note that the parameter $\beta$ in (15) does not need to be chosen: it is sufficient to consider a set of values reasonable for this parameter; then, selection of the most suitable

\begin{tabular}{|c||c|c|}
\hline & $D^{I}$ & $D^{V}$ \\
\hline \hline$F I T$ & $87 \%$ & $85 \%$ \\
\hline
\end{tabular}

Table 1. FIT indexes obtained by the BIM model in simulation on the identification and validation sets.

parameter values is performed by algorithm (12), thanks to minimization of the signal coefficient $\ell_{1}$-norm. Once the basis functions have been defined, the value $\varepsilon=58$ has been taken for the noise bound in (12). This value has been chosen by means of a trial and error procedure on the identification set. The CVX toolbox Grant and Boyd (2010) has been used to solve the optimization problem (12). The identified model is described by the difference equation (13), where $\hat{f}$ and $\hat{d}_{k}$ are given by (9).

The BIM model has been first tested on the identification set $D^{I}$. A simulation of the model has been performed, using as inputs the insulin signal described above and the disturbance $\hat{d}_{k}$ estimated by algorithm (12). The FIT index has been considered to evaluate the model performance. This index measures the percentage of data that are correctly explained by the model, and is defined as

$$
F I T \doteq 100\left(1-\frac{\|y-\hat{y}\|_{2}}{\|y-\operatorname{mean}(y)\|_{2}}\right) \%
$$

where $y$ and $\hat{y}$ are the measured output signal and the one simulated by the model, respectively. The FIT value obtained in the simulation is shown in Table 1.

Then, the BIM model has been tested on the validation set $D^{V}$. A simulation of the model has been performed, using as inputs the insulin signal and the disturbance $\hat{d}_{k}$ estimated on the validation set, solving the optimization problem (12) only with respect to $b$, with $a$ fixed and equal to the vector previously estimated on the identification set. The FIT value obtained in the simulation is shown in Table 1 . The output simulated by the model is compared to the measured one in Figure 2. The estimated disturbance $\hat{d}_{k}$ is compared to the meal input in Figure 3 for a portion of the validation set.

From these results, it can be concluded that the identified BIM model is able to reproduce quite accurately the behavior of the "true" system. Moreover, the recovered disturbance $\hat{d}_{k}$ correctly describes the unmeasured meal input, since it provides a quantitative information on the times when the meals have occurred and on the entity of these meals. We remind that $\hat{d}_{k}$ is not an estimate of the unmeasured input $w_{k}$ but a surrogate of it, describing the effect of $w_{k}$ on the system output.

\subsection{Identification of prediction models}

Besides simulation, models are important in the diabetes context for obtaining accurate predictions of the blood glucose concentration, since these predictions can be effectively used e.g. for therapy decision or predictive control design. Thus, a prediction time horizon of half an hour, corresponding to 6 steps with the used sampling time of 5 minutes, has been considered, since this time horizon can be of interest for predictive control. Considering the identification data set, a model and a disturbance signal have been estimated by means of algorithm (12). While in Section 3.2, the disturbance has been recovered in an anticausal way (to obtain the estimate $\hat{d}_{k}$ at time $k$, the past, current and future data $\left(\tilde{y}_{l}, \tilde{r}_{l}\right)$, with $l=1, \ldots, k, \ldots L$ 


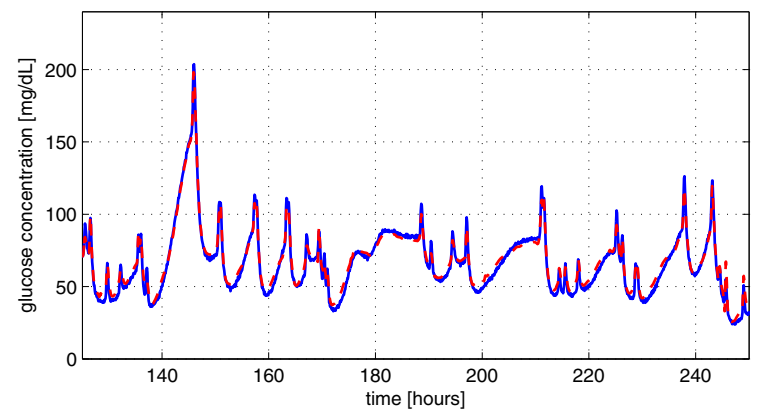

Fig. 2. BIM model simulation (validation set). Continuous (blue) line: measured output. Dashed (red) line: BIM model simulation.

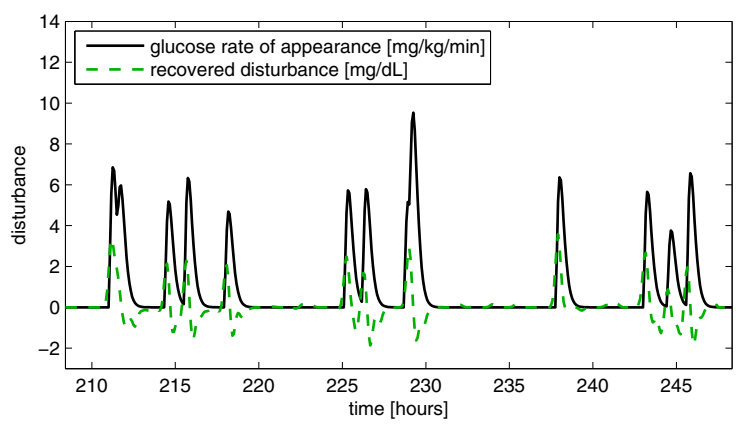

Fig. 3. Disturbance recovering (validation set). Continuous (black) line: glucose rate of appearance in the blood (unknown). Dashed (green) line: recovered disturbance.

have been used), here a causal estimate of the disturbance has been obtained (only the past and current data $\left(\tilde{y}_{l}, \tilde{r}_{l}\right)$, with $l \leq k$, have been used to estimate $\hat{d}_{k}$ ). Next, using the identified model and solving the optimization problem (12) only with respect to $b$, a causal estimate of the disturbance has been obtained also for the validation set. Then, a prediction model, called BIP, has been estimated from the identification set using the Matlab System Identification Toolbox, considering as inputs the insulin signal and the causally recovered disturbance. In particular, an ARMAX model of order 5 has been obtained from this identification. Note that the model originally identified together with the disturbance may be very accurate in simulation but is not suitable for prediction, since one of its inputs is the anti-causally recovered disturbance and this signal clearly cannot be used for prediction.

The BIP model has been tested in prediction on the validation set $D^{V}$. The FIT index obtained by the model is reported in Table 2. Note that, solving the optimization problem (12) with the setting considered here requires about 20 seconds on an average personal computer (without using ad-hoc algorithms). Thus, online optimization can be reasonably performed in the case of a real diabetes patient, since model predictive control requires significantly longer sampling times for this application.

For comparison, several ARX, ARMAX and OE linear models and several NARX nonlinear models of order 5 have been estimated on the identification set $D^{I}$, using the Matlab System Identification Toolbox, following a "standard" approach (i.e. considering as input only the insulin signal and not the recovered disturbance). Most of the "standard" models showed negative FIT indexes or even input-output (IO) instability in simulation both on

\begin{tabular}{|c||c|c|c|}
\hline & BIP & NARX & ARX \\
\hline \hline$F I T$ & $78 \%$ & $71 \%$ & $52 \%$ \\
\hline
\end{tabular}

Table 2. FIT indexes obtained by the best models in 6-step ahead prediction on the validation set.

the identification and validation sets. Nevertheless, some of these models displayed a good performance in 6-step ahead prediction. In Table 2, the FIT indexes obtained on the validation set $D^{V}$ by the best linear and nonlinear models are reported.

From these prediction results, it can be concluded that, thanks to the utilization of the (causally) recovered disturbance, the BIM model can achieve better accuracies than other models obtained using "standard" approaches.

\section{CONCLUSIONS}

In this paper, a novel black-box model identification approach has been proposed to derive accurate models of glucose control in Type 1 diabetic patients. This approach, allows us to recover a signal describing the effect of all unknown (or not easily measurable) inputs acting on the patient system (e.g. meal input, physical activity, emotions, etc. ). Simulations have been performed to show and confirm the advantages in using this new approach. The identified model has been used to simulate the behavior of the patient system, taking realistically into account the effects of the unknown external inputs. Moreover, using a model and a reliable estimate of the unknown external inputs allowed us to obtain improved prediction performances with respect to "standard" prediction approaches.

\section{REFERENCES}

K. Abed-Meraim, W. Qiu, and Y. Hua. Blind system identification. Proceedings of the IEEE, 85(8):1310$1322,1997$.

Ishan Ajmera, Maciej Swat, Camille Laibe, Nicolas Le Novère, and Vijayalakshmi Chelliah. The impact of mathematical modeling on the understanding of diabetes and related complications. CPT: Pharmacometrics $\&$ Systems Pharmacology, 2:e54, 2013.

E.W. Bai and F. Minyue. A blind approach to hammerstein model identification. IEEE Transactions on Signal Processing, 50(7):1610-1619, 2002. ISSN 1053587X. doi: 10.1109/TSP.2002.1011202.

B. Wayne Bequette. Challenges and recent progress in the development of a closed-loop artificial pancreas. Annual Reviews in Control, 36(2):255 - 266, 2012. ISSN 1367-5788. doi: http://dx.doi.org/10.1016/j.arcontrol.2012.09.007.

R N Bergman, L S Phillips, and C Cobelli. Physiologic evaluation of factors controlling glucose tolerance in man: measurement of insulin sensitivity and beta-cell glucose sensitivity from the response to intravenous glucose. The Journal of Clinical Investigation, 68(6): 1456-1467, 12 1981. doi: 10.1172/JCI110398.

E.J. Candes and T. Tao. Near-optimal signal recovery from random projections: Universal encoding strategies? IEEE Transactions on Information Theory, 52 (12):5406 -5425, dec. 2006. ISSN 0018-9448. doi: 10.1109 /TIT.2006.885507.

C. Cobelli, C. Dalla Man, G. Sparacino, L. Magni, G. De Nicolao, and B.P. Kovatchev. Diabetes: Models, signals, and control. IEEE Reviews in Biomedical Engineering, 2:54-96, 2009. ISSN 1937-3333. doi: 10.1109/RBME.2009.2036073. 
C. Cobelli, E. Renard, and B. Kovatchev. Artificial pancreas: Past, present, future. Diabetes, 60:2672-2682, 2011.

D.L. Donoho, M. Elad, and V.N. Temlyakov. Stable recovery of sparse overcomplete representations in the presence of noise. IEEE Transactions on Information Theory, 52(1):6 - 18, jan. 2006. ISSN 0018-9448. doi: 10.1109/TIT.2005.860430.

S. Dube, I. Errazuriz, C. Cobelli, R. Basu, and A. Basu. Assessment of insulin action on carbohydrate metabolism: Physiological and non-physiological methods. Diabetic Medicine, 30(6):664-670, 2013.

D.A. Finan, F.J. Doyle 3rd., C.C. Palerm, W.C. Bevier, H.C. Zisser, L. Jovanovic, and D.E. Seborg. Experimental evaluation of a recursive model identification technique for type 1 diabetes. Journal of diabetes science and technology, 3(5):1192-1202, 2009. cited By (since 1996)11.

J.J. Fuchs. Recovery of exact sparse representations in the presence of bounded noise. IEEE Transactions on Information Theory, 51(10):3601 -3608, oct. 2005. ISSN 0018-9448. doi: 10.1109/TIT.2005.855614.

M.A. Gonzalez-Olvera, A.G. Gallardo-Hernndez, Y. Tang, M.C. Revilla-Monsalve, and S. Islas-Andrade. A continuous-time recurrent neurofuzzy network for blackbox modeling of insulin dynamics in diabetic type-1 patients. In Wen $\mathrm{Yu}$ and EdgarN. Sanchez, editors, Advances in Computational Intelligence, volume 116 of Advances in Intelligent and Soft Computing, pages 219228. Springer Berlin Heidelberg, 2009. ISBN 978-3-64203155-7.

M. Grant and S. Boyd. CVX: Matlab software for disciplined convex programming, version 1.21 . http://cvxr.com/cvx, August 2010.

R.A. Harvey, Youqing Wang, B. Grosman, M.W. Percival, W. Bevier, D.A. Finan, H. Zisser, D.E. Seborg, L. Jovanovic, F.J. Doyle, and E. Dassau. Quest for the artificial pancreas: Combining technology with treatment. Engineering in Medicine and Biology Magazine, IEEE, 29(2):53-62, 2010.

N. Kalouptsidis, P. Koukoulas, and V.J. Mathews. Blind system identification. IEEE Transacions on signal processing, 51(2):484-499, 2003.

Joon Bok Lee, E. Dassau, D.E. Seborg, and F.J. Doyle. Model-based personalization scheme of an artificial pancreas for type 1 diabetes applications. In American Control Conference (ACC), 2013, pages 2911-2916, June 2013.

M. Milanese and A. Vicino. Optimal algorithms estimation theory for dynamic systems with set membership uncertainty: an overview. Automatica, 27:997-1009, 1991.

G. Mileounis and N. Kalouptsidis. Blind identification of second order volterra systems with complex random inputs using higher order cumulants. IEEE Transactions on Signal Processing, 57(10):4129-4135, 2009. ISSN 1053-587X.

C. Novara. Sparse identification of nonlinear functions and parametric set membership optimality analysis. In American Control Conference, San Francisco, USA, 2011.

C. Novara. Sparse identification of nonlinear functions and parametric set membership optimality analysis. IEEE Transactions on Automatic Control, 57(12):3236-3241, 2012. doi: 10.1109/TAC.2012.2202051.

Gianluca Nucci and Claudio Cobelli. Models of subcutaneous insulin kinetics. a critical review. Computer Methods and Programs in Biomedicine, 62(3):249 - 257, 2000. ISSN 0169-2607. doi: http://dx.doi.org/10.1016/S01692607(00)00071-7.
H. Ohlsson, L. Ratliff, R. Dong, and S.S. Sastry. Blind identification of arx models with piecewise constant inputs. In arXiv:1303.6719 [cs.SY], 2013.

E. Renard, C. Cobelli, and B.P. Kovatchev. Closed loop developments to improve glucose control at home (in press). Diabetes Research and Clinical Practice, 2013.

J. Sjöberg, Q. Zhang, L. Ljung, A. Benveniste, B.Delyon, P. Glorennec, H. Hjalmarsson, and A. Juditsky. Nonlinear black-box modeling in system identification: a unified overview. Automatica, 31:1691-1723, 1995.

F. Stahl and R. Johansson. Diabetes mellitus modeling and short-term prediction based on blood glucose measurements. Mathematical Biosciences, 217(2):101 - 117, 2009. ISSN 0025-5564.

J.A. Tropp. Just relax: convex programming methods for identifying sparse signals in noise. IEEE Transactions on Information Theory, 52(3):1030 -1051, mar. 2006. ISSN 0018-9448. doi: 10.1109/TIT.2005.864420.

T. L. Vincent and C. Novara. Mixed parametric/nonparametric identification of systems with discontinuous nonlinearities. Automatica, 49(12): $3661-3669,2013$. ISSN 0005-1098. doi: http://dx.doi.org/10.1016/j.automatica.2013.09.022.

A. Wills, T.B. Schon, L. Ljung, and B. Ninnes. Blind identification of wiener models. In 18th IFAC World Congress, Milano, Italy, 2011. 